\title{
Effet des Variables Agro-Écologiques sur la Distribution de la Végétation de la Rôneraie Anthropisée de Dasga au Niger
}

\author{
B. Katkoré, \\ M. Moussa, \\ Institut National de la Recherche Agronomique du Niger \\ (INRAN), Maradi, Niger \\ I. Soumana, \\ A. Mahamane, \\ Département de Biologie, Faculté des Sciences, \\ Université Abdou Moumouni (UAM), Niamey \\ T. Abasse, \\ Institut National de la Recherche Agronomique du Niger \\ (INRAN), Maradi, Niger \\ M. Larwanou, \\ International Crops Research Institute for the Semi-Arid Tropics \\ (ICRISAT), Niamey, Niger
}

Doi: 10.19044/esj.2019.v15n3p406 URL:http://dx.doi.org/10.19044/esj.2019.v15n3p406

Résumé

Cette étude a pour objectif de contribuer à la gestion durable de la rôneraie de Dasga à travers l'analyse de la phytodiversité et des paramètres écologiques influençant la distribution de sa végétation. Les méthodes de relevé phytosociologique et d'analyses multivariées ont été utilisées. Les relevés de la végétation ont été effectués selon l'approche sigmatiste de Braun-Blanquet (1932) et les techniques d'analyses multivariées employées ont été celles relatives à l'analyse factorielle des correspondances détendancées (DCA) et l'analyse canonique des correspondances (CCA). Au total 144 espèces réparties dans 100 genres et 50 familles ont été recensées à partir de 68 relevés. Cette flore a été dominée par une forte présence des thérophytes et d'espèces à large distribution pantropicale et paléotropicale, montrant ainsi un environnement aride et perturbé. Les analyses multivariées (DCA et CCA) ont permis d'identifier les gradients de distribution des plantes. Parmi les variables testées, seules la pente et les cultures se sont revelées déterminantes dans la structuration de la végétation par le test de permutation de Monte Carlo. Ces variables agro-écologiques sembleraient 
être les paramètres majeurs de la distribution des espèces végétales dans cette rôneraie. Ainsi la compréhension des relations entre la végétation et les paramètres écologiques peut être utilisée comme outil d'aménagement durable des rôneraies au Niger.

Mots clés: Phytodiversité, distribution, variables agro-écologiques, analyse multivariée, rôneraie anthropisée, Niger

\title{
Effect of Agro-Ecological Variables on Vegetation Distribution in the Disturbing Palm Trees Forest of Dasga in Niger
}

\author{
B. Katkoré, \\ M. Moussa, \\ Institut National de la Recherche Agronomique du Niger \\ (INRAN), Maradi, Niger

\section{Soumana,} \\ A. Mahamane, \\ Département de Biologie, Faculté des Sciences, \\ Université Abdou Moumouni (UAM), Niamey \\ T. Abasse, \\ Institut National de la Recherche Agronomique du Niger \\ (INRAN), Maradi, Niger \\ M. Larwanou, \\ International Crops Research Institute for the Semi-Arid Tropics \\ (ICRISAT), Niamey, Niger
}

\begin{abstract}
The objective of this study is to contribute to the sustainable management of the Dasga palm trees forest by analyzing the phytodiversity and ecological parameters guiding the distribution of vegetation. The methods of phytosociological survey and multivariate analyzes were used. The vegetation surveys were carried out according to the sigmatistic approach of Braun-Blanquet (1932) and the multivariate analysis techniques used were those relating to factorial analysis of relaxed correspondence (DCA) and canonical correspondence analysis (CCA). A total of 144 species belonging to 100 genera and 50 families were recorded from 68 plots. This flora is
\end{abstract}


dominated by a strong presence of therophytes and species with broad pantropical and palaeotropical distribution, thus showing an arid and perturbed environment. The multivariate analyzes (DCA and CCA) sow among the variables tested; only the slope and the crops were determinants in the structuring of the vegetation by the Monte Carlo permutation test. These ecological variables would appear to be the major parameters of species distribution. Thus the understanding of the relationships between vegetation and ecological parameters can be used as a tool for sustainable management of this palm trees forest.

Keywords: Phytodiversity, distribution, agro-ecological variables, multivariate analysis, palm trees, Niger

\section{Introduction}

La rôneraie de Dasga est située dans le canton de Kantché, elle est la seule forêt classée de la commune de Matamèye depuis 1942 (PAPAGE, 2004). La rôneraie de Dasga est exploitée par trois ethnies notamment les Haoussas, les Touaregs et les Peulhs regroupés en 1000 habitants et 58 ménages avec 450 femmes, 300 hommes et 250 enfants tous sexes confondus (Katkoré, 2011). Suite aux demandes accrues en terres de cultures, conséquence d'une croissance démographique, une partie de la rôneraie a été déclassée. Les terres ont été temporairement accordées aux populations conformément aux dispositions législatives en vigueur dans le pays. La rôneraie a subi une fragmentation avec la formation des enclaves pastorales, des corridors, des couloirs de passage et des parcs agroforestiers. La rôneraie de Dasga joue des rôles socioéconomiques importants pour les populations. En effet, elle assure la zone de transhumance et de pâturage pour les animaux et contribue à près de $65 \%$ aux bois de chauffage, $60 \%$ bois d'œuvre et $55 \%$ bois de service pour les populations (Katkoré, 2011). En général, au Niger les besoins fondamentaux des populations rurales sont satisfaits à $90 \%$ à partir de la biodiversité (CNEDD, 1998). Les études sur la biodiversité, menées jusqu'ici restent fragmentaires ou générales (Baina, 2000). De même, la description des écosystèmes et des groupements végétaux n'est pas encore effective ou actualisée. Des telles dépendances vis-à-vis de la biodiversité conduisent à la dégradation des écosystèmes forestiers. Des nombreuses études dans la bande sud du pays ont montré une dégradation des écosystèmes forestiers suite aux changements importants dans l'occupation des sols, à la surexploitation des ressources naturelles et aux sécheresses des dernières années (Mahamane et al., 2007 ; Issiaka et al., 2012 ; Hamidou et al., 2012 ; Sadda et al., 2016). Cette dégradation a conduit par endroit à la raréfaction et à la disparition de nombreuses espèces ligneuses des écosystèmes (Larwanou and Saâdou, 2005 ; Moussa et $a l ., 2016)$. Force est de constater que dans la 
rôneraie de Dasga, il est difficile de noter des initiatives spontanées tendant à protéger l'écosystème en général et les espèces végétales en particulier. Il urge de renforcer les efforts de conservation et de préservation de toutes les espèces mais aussi de mise en valeur efficiente des ressources disponibles. Pour cela, la connaissance de cet écosystème tant dans sa diversité, les facteurs influençant sa dynamique et son fonctionnement que dans sa contribution socioéconomique et écologique pour le milieu, semble nécessaire en vue d'une meilleure formulation des politiques d'aménagement. Cette étude s'inscrit dans une telle dynamique, en abordant la connaissance et les effets des variables agro-écologiques notamment les formations géomorphologiques et les cultures sur la distribution de la biodiversité dans la rôneraie de Dasga. Elle a pour objectif de contribuer à la gestion durable de cette rôneraie en combinant les relevés phyto-sociologiques aux analyses multivariées en vue d'analyser : (i) sa phyto-diversité et (ii) les paramètres agro-écologiques guidant la distribution de la végétation.

\section{Matériel et méthodes}

\section{Zone d'étude}

Cette étude a été réalisée dans le centre-est du Niger de la région de Zinder (Figure 1). Le climat de la zone est typiquement tropical sec avec une pluviométrie moyenne annuelle de 400 à $600 \mathrm{~mm}$ du Nord au Sud, répartie entre juin et septembre. Le mois le plus frais est Janvier avec une température moyenne mensuelle de $19^{\circ} \mathrm{C}$ et le plus chaud est Mai avec une moyenne de $32^{\circ} \mathrm{C}$. Le substrat édaphique est du sable dunaire du continental hamadien d'âge quaternaire (Saâdou, 1990). Dans ces conditions climatiques et écologiques, le développement et la distribution de la végétation sont liés à la répartition des pluies dans le temps et dans l'espace (Saâdou, 1990). Ainsi, sur les plateaux latéritiques, on a des fourrés à combrétacées, des savanes sur les terrasses sableuses méridionales et des steppes sur les dunes et dans les vallées sèches. 


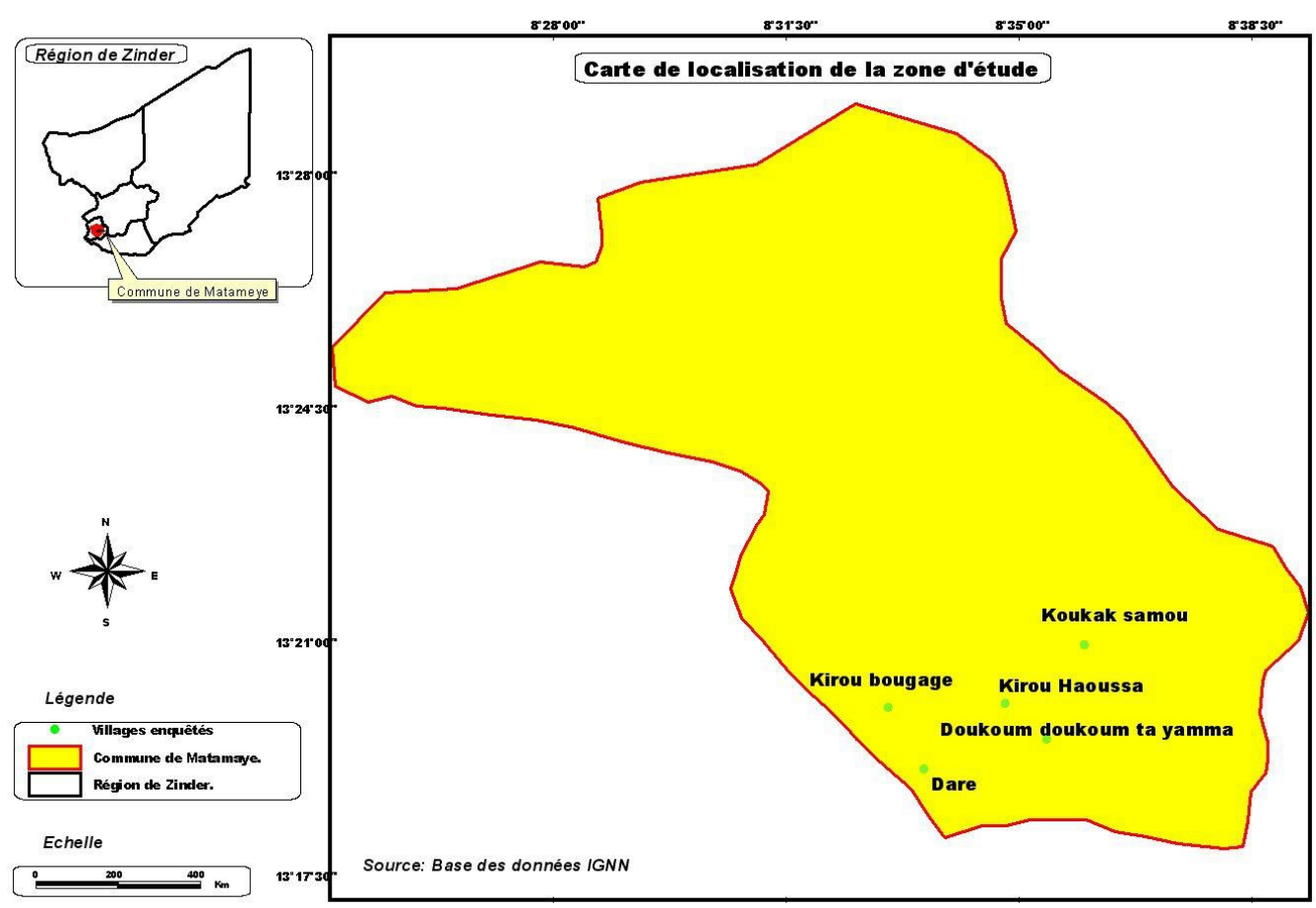

Figure 1: Carte de localisation de la zone d'étude

\section{Méthodes}

L'échantillonnage s'est basé sur les critères bio-physionomiques et mésologiques obtenus à partir des images satellitaires de la zone. Ces images ont été traitées avec le logiciel Arcview GIS 3.2 et Envi 4.2 selon l'approche utilisée par Mahamane et al (2007). Il s'agit de : l'acquisition du support cartographique, la correction géométrique, la répartition de la zone d'étude à l'aide des images landsat, la mission de reconnaissance et vérité terrain, les relevés des coordonnées des points et leur codification, l'interprétation des images, la correction et la finalisation des cartes ont permis de déterminer quatre unités d'occupations des sols à savoir les zones de cultures, les unités de la forêt classée, la zone de pâturage et le couloir de passage (Figure 2). Le dispositif de sondage est du type systématique. Les collectes de données de végétation relatives à cette étude ont été réalisées entre septembre et octobre, période correspondant à l'optimum de croissance de la végétation au Sahel (Boudet, 1984). Il est conçu en tenant compte de la géomorphologie, la topographie et le type du substrat, suivant un gradient pluviométrique nordsud et surtout des unités d'occupation des sols. Un total de 12 transects latitudinaux distant de $500 \mathrm{~m}$ ont été tracés. Sur chaque transect ont été placées des placettes rectangulaires de $1000 \mathrm{~m}^{2}(20 \mathrm{~m}$ x $50 \mathrm{~m})$ distant de $250 \mathrm{~m}$ (Thombiano et al., 2015). Au total, 68 relevés phyto-sociologiques ont été 
effectués dans ces placettes selon la méthode sigmatiste de Braun-Blanquet (1932). Cette méthode s'est distinguée comme étant la technique la plus utilisée dans l'étude de la végétation en Afrique tropicale présente comme avantage de dresser la liste floristique de la zone investiguée et d'appréhender les conditions du milieu par l'intermédiaire des espèces (Gounot, 1969). Lors des relevés sur une fiche normalisée, ont été notés, les paramètres stationnels (coordonnées géographiques, texture du sol par la méthode tactile, topographie, géomorphologie) et les coefficients d'abondance-dominance de toutes les espèces végétales rencontrées au moyen de critères suivants :

5 : espèce recouvrant plus de $75 \%$ de la surface du relevé

4 : espèce recouvrant entre $50 \%$ et $75 \%$ de la surface du relevé

3 : espèce recouvrant entre $25 \%$ et $50 \%$ de la surface du relevé

2 : espèce recouvrant entre $5 \%$ et $25 \%$ du relevé

1 : espèce abondante à peu abondante et recouvrant moins de $5 \%$ de la surface du relevé

+ : espèce rare et recouvrant moins de $1 \%$ de la surface du relevé.

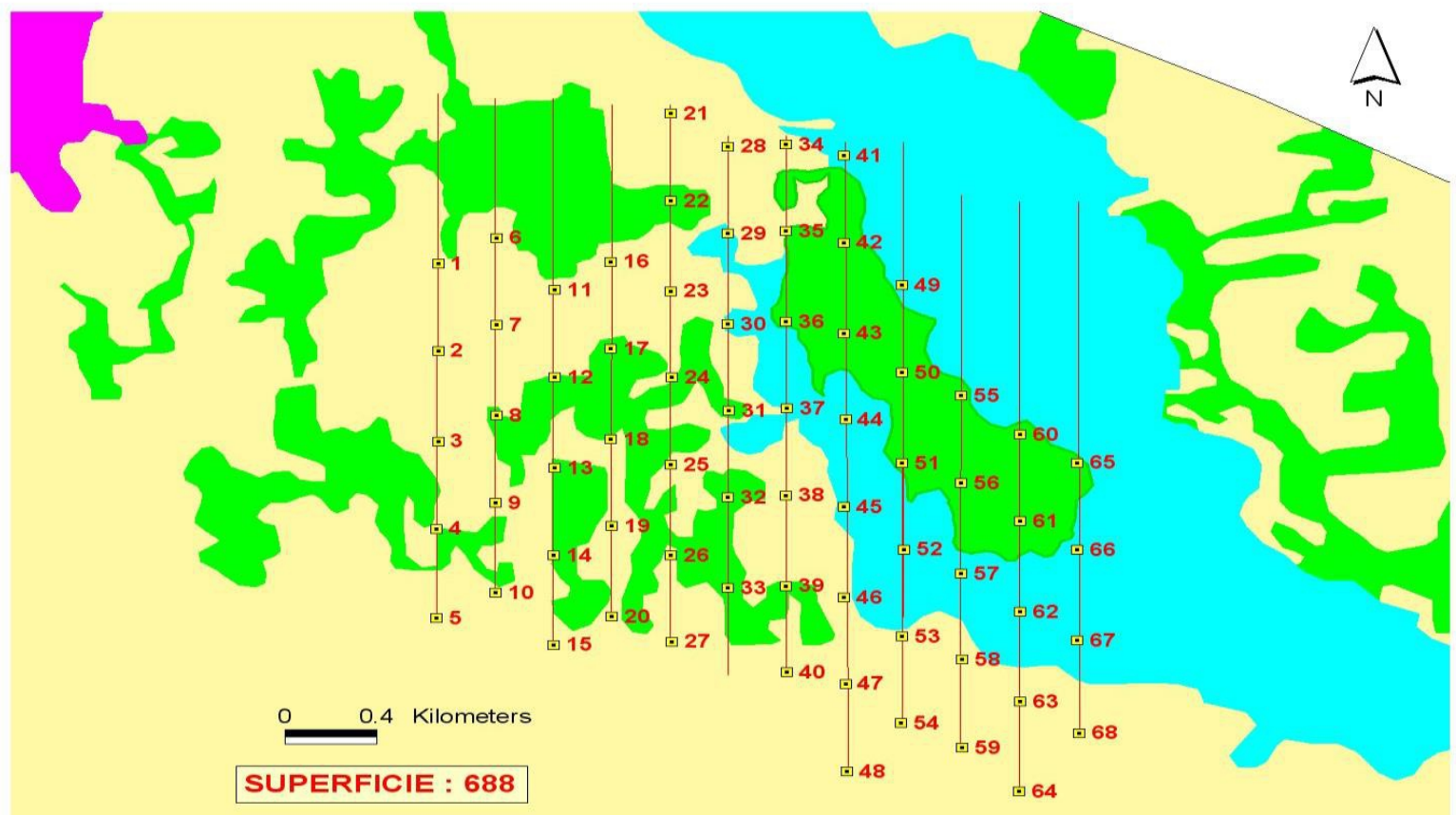

Figure 2 : Illustration des techniques d'échantillonnages et de dispositions des placettes de relevés

Carte établie à l'aides images Landsat ETM+ de 2002, base de données IGNN et des donnés de terrain 2009. 


\section{Analyses des données}

\section{Analyses écologiques et chorologiques}

Les spectres écologiques sont celles partagés par des nombreuses études (Oumarou, 2003 et Mahamane, 2005). Ainsi on distingue : le spectre brut qui indique la proportion centésimale des espèces appartenant à chaque catégorie considérée et le spectre pondéré qui est calculé en attribuant à chaque espèce un intervalle de valeur correspondant à son coefficient d'abondancedominance pour l'ensemble des relevés contenant les espèces de la catégorie considérée. Ainsi on a $:(+)=0,5 ;(1)=3 ;(2)=15 ;(3)=37,5 ;(4)=62,5$ et $(5)=87,5$. Pour la détermination des formes biologiques, le système de Raunkiaer (1934) a été utilisé. Pour ce qui est de la détermination des types phytogéographiques, les subdivisons chorologiques pour l'Afrique de White (1983) adoptées par (Saâdou, 1990) ont été utilisées.

\section{Analyse des données phytoécologiques}

\section{Analyse factorielle des correspondances}

L'ordination des relevés a été abordée par les techniques d'analyses multivariées. Il s'agit ici de l'Analyse Factorielle des Correspondances Détendancées (DCA). Cette méthode a été réalisée à l'aide du logiciel CANOCO (Canocal Community Ordination) for Windows, version 3.1 (Ter Braak et Smilauer, 1998). Ainsi, une matrice de 68 relevés et 144 espèces a été soumise à l'AFC pour l'ordination des relevés. L'analyse est basée sur des tableaux floristiques et permet de réduire la variabilité floristique entre relevés à un nombre réduit (deux ou trois en général) d'axes factoriels (Mahamane, 2005).

\section{Croisement entre descripteurs floristique et écologique}

Les relations végétation-environnement ont été mises en évidence par l'Analyse Canonique des Correspondances (ACC). Elle procède comme l'analyse factorielle des correspondances et elle tend à maximiser les distances entre les optimums écologiques des espèces. Elle s'arrange toutefois pour que l'ordination des relevés soit la meilleure combinaison linéaire possible avec des variables écologiques. La mise en relation permet d'évaluer la proportion de la variabilité floristique pouvant être expliquée par les variables environnementales. Ainsi, une matrice de 68 relevés croisés aux variables environnementales a été soumise à l'ACC.

Les variables étudiées se rapportent plus particulièrement à la topographie, la géomorphologie, aux types d'usages et à la texture du sol. Ces variables et modalités sont réparties comme suit :

- Topographie (2 modalités : pente moyenne et faible) ;

- Géomorphologie (2 modalités : vallée et terrasse sableuse);

- Texture (2 modalités : sable et limon-sable); 
- Usages (4 modalités : champs, forêt, zone pastorale et couloir de passage).

La nomenclature des groupements végétaux identifiés a été effectuée sur la base de la dominance des espèces (Roussel, 1987 et Fournier, 2000). En effet le nom d'un groupement découle de deux espèces ayant le plus fort recouvrement moyen.

\section{Résultats}

\section{Phytodiversité, phytogéographie et types biologiques}

Les 68 relevés de végétation menés dans la rôneraie de Dasga ont permis de recenser 144 espèces végétales réparties dans 100 genres et 50 familles (Tableau 1). Le nombre d'espèces par relevé varie de 6 à 12 avec une moyenne de 7,61. Les familles les plus représentées sont: Poaceae (16 espèces, soit 11,11\%), Fabaceae (11 espèces soit 7,64 \%), Mimosaceae (10 espèces soit 6,94 \%), Caesalpiniaceae (8 espèces soit 5,55\%), Convolvilaceae ( 7 espèces soit 4,86 \%) et Anacardiaceae (6 espèces soit 4,17 $\%)$. Pour les genres, les familles les mieux représentées sont : Poaceae (10 genres soit $10 \%$ ), Fabaceae (6 genres soit $6 \%$ ), Mimosaceae, Rubiaceae, Euphorbiaceae et Capparidaceae (4 genres soit $4 \%$ ) (Tableau 1).

Tableau 1 : Nombre et pourcentage d'espèces et de genres par famille

\begin{tabular}{lllll}
\hline Familles & $\begin{array}{l}\text { Genres } \\
\text { (Nombre) }\end{array}$ & \% & $\begin{array}{l}\text { Espèces } \\
\text { (Nombre) }\end{array}$ & $\%$ \\
\hline Poaceae & 10 & 10 & 16 & 11,11 \\
Fabaceae & 6 & 6 & 11 & 7,64 \\
Mimosaceae & 4 & 4 & 10 & 6,94 \\
Caesalpiniaceae & 3 & 3 & 8 & 5,55 \\
Convolvilaceae & 2 & 2 & 7 & 4,86 \\
Anacardiaceae & 3 & 3 & 6 & 4,17 \\
Asteraceae & 2 & 2 & 5 & 3,47 \\
Cyperaceae & 3 & 3 & 5 & 3,47 \\
Rubiaceae & 4 & 4 & 5 & 3,47 \\
Asclepiadaceae & 3 & 3 & 4 & 2,78 \\
Euphorbiaceae & 4 & 4 & 4 & 2,78 \\
Capparidaceae & 4 & 4 & 4 & 2,78 \\
Amaranthaceae & 3 & 3 & 4 & 2,78 \\
Aizoaceae & 3 & 3 & 4 & 2,78 \\
Arecaceae & 3 & 3 & 3 & 2,09 \\
Moraceae & 3 & 3 & 3 & 2,09 \\
Combretaceae & 3 & 3 & 3 & 2,09 \\
Malvaceae & 2 & 2 & 3 & 2,09 \\
Tiliceae & 2 & 2 & 3 & 2,09 \\
Cucurbitaceae & 3 & 3 & 3 & 2,09 \\
Solanaceae & 2 & 2 & 2 & 1,39 \\
Commelinaceae & 1 & 1 & 2 & 1,39 \\
Bombacaceae & 2 & 2 & 2 & 1,39 \\
Myrtaceae & 2 & 2 & 2 & 1,39
\end{tabular}




\begin{tabular}{lllll} 
Rhamnaceae & 1 & 1 & 2 & 1,39 \\
Pedaliaceae & 2 & 2 & 2 & 1,39 \\
Lamiaceae & 2 & 2 & 2 & 1,39 \\
Scrophulariaceae & 2 & 2 & 2 & 1,39 \\
Caryophyllaceae & 2 & 2 & 2 & 1,39 \\
Sterculariaceae & 2 & 2 & 2 & 1,39 \\
Acanthaceae & 1 & 1 & 2 & 1,39 \\
Nyctaginaceae & 1 & 1 & 1 & 0,69 \\
Polygalaceae & 1 & 1 & 1 & 0,69 \\
Portulacaceae & 1 & 1 & 1 & 0,69 \\
Verbenaceae & 1 & 1 & 1 & 0,69 \\
Ebenaceae & 1 & 1 & 1 & 0,69 \\
Lythraceae & 1 & 1 & 1 & 0,69 \\
Rosaceae & 1 & 1 & 1 & 0,69 \\
Balanitaceae & 1 & 1 & 1 & 0,69 \\
Moringaceae & 1 & 1 & 1 & 0,69 \\
Burseraceae & 1 & 1 & 1 & 0,69 \\
Annonaceae & 1 & 1 & 1 & 0,69 \\
\hline Total & $\mathbf{1 0 0}$ & $\mathbf{1 0 0}$ & $\mathbf{1 4 4}$ & $\mathbf{1 0 0}$ \\
\hline
\end{tabular}

Les formes de vie les plus abondantes ont été les thérophytes $(\mathrm{T})$ avec 65 espèces (45,14\%), les microphanérophytes (Mcp) avec 37 espèces $(25,69 \%)$ et les mésophanérophytes (Msp) avec 11 espèces $(7,64 \%)$. Les géophytes rhizomateux (Gr), les nanophanérophytes (Np), les mégaphanérophytes (Mpfe) et les hydrophytes (Hy) ont été très peu représentés dans cette rôneraie avec chacune de forme, 0,69 \% (Figure 3). Sur le plan chorologique, cette flore est caractérisée par une prédominance des espèces Soudano-Zambésiennes avec 47 espèces (32,64 \%), suivies des Guinéo-Congolaises-Soudano-Zambésiennes avec 40 espèces $(27,78 \%)$ et des Soudano-Zambéziennes-Saharosindiennes avec 34 espèces $(23,62 \%)$. 


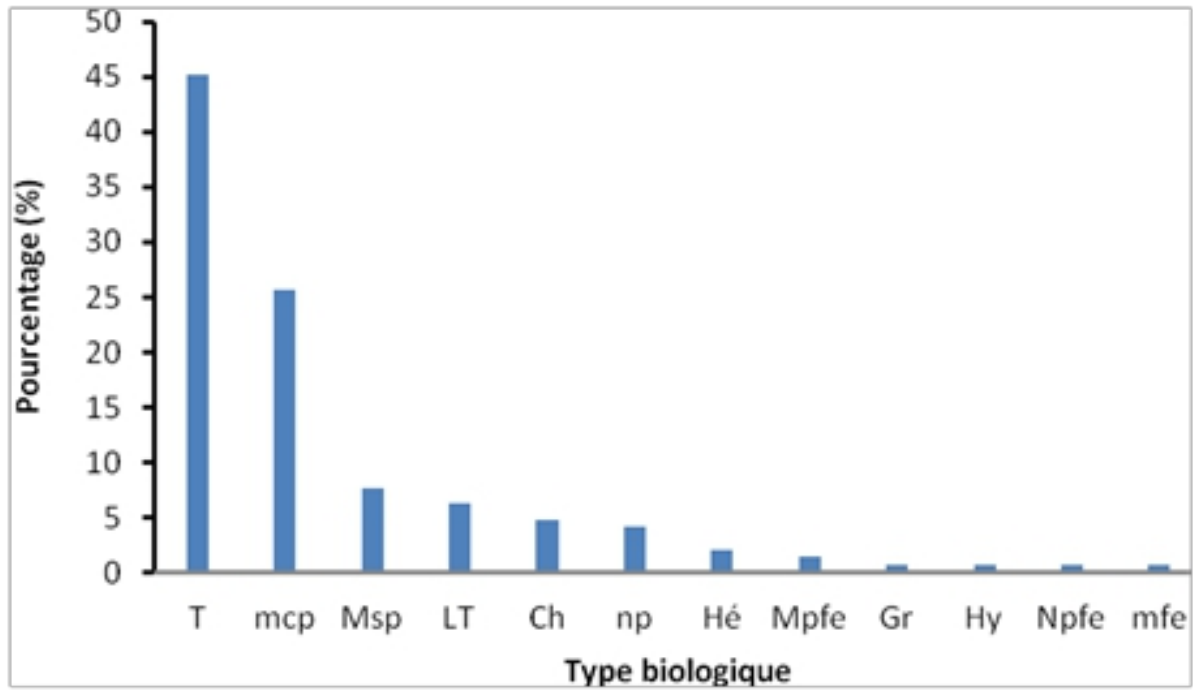

Figure 3 : Spectre global des formes biologiques

T: thérophytes, Mcp : microphanérophytes, Msp : mésophanérophytes, Lt: lianes thérophytes, $\mathrm{Ch}$ : chaméphytes, $\mathrm{Np}$ : nanophanérophytes, Hé : Hémicryptophytes, Gr : géophytes rhizomateux, Hy : hydrophytes

L'importance numérique des éléments à large distribution montre que la flore de la rôneraie de Dasga se caractérise par l'abondance d'espèces étrangères. Parmi les 144 espèces recensées, $52(36,11 \%)$ sont des ligneux et 92 herbacées $(63,89 \%)$. Les ligneux les plus dominants sont Borassus aethiopum (43,5\%), Hyhaene thebaica (15,1\%), Faidherbia albida $(11,63 \%)$, Lannea microcarpa $(4,81 \%)$ et les herbacées dominantes sont Eragrostis tremula $(23,65 \%)$ et Alysicarpus ovalifolius $(17,62)$. D'autres espèces étrangères la zone ont été recensées notamment Azadirachta indica, Moringa oleifera, Eucalyptus camaldulensis, Prosopis juliflora, Anacardium occidentale, Mangifera indica, Psidium guajava.

\section{Analyse indirecte du gradient de distribution des espèces (DCA)}

La matrice des 68 relevés et 144 espèces a été soumises à une Analyse Factorielle de Correspondance Détendancée (DCA) (Figure 4). Les axes 1 et 2 de la carte factorielle expliquent respectivement $11,4 \%$ et $6,9 \%$ la dispersion des informations sur le plan de l'ordination. Le DCA montre que nos relevés sont répartis de manière homogène sur le plan de l'ordination. 


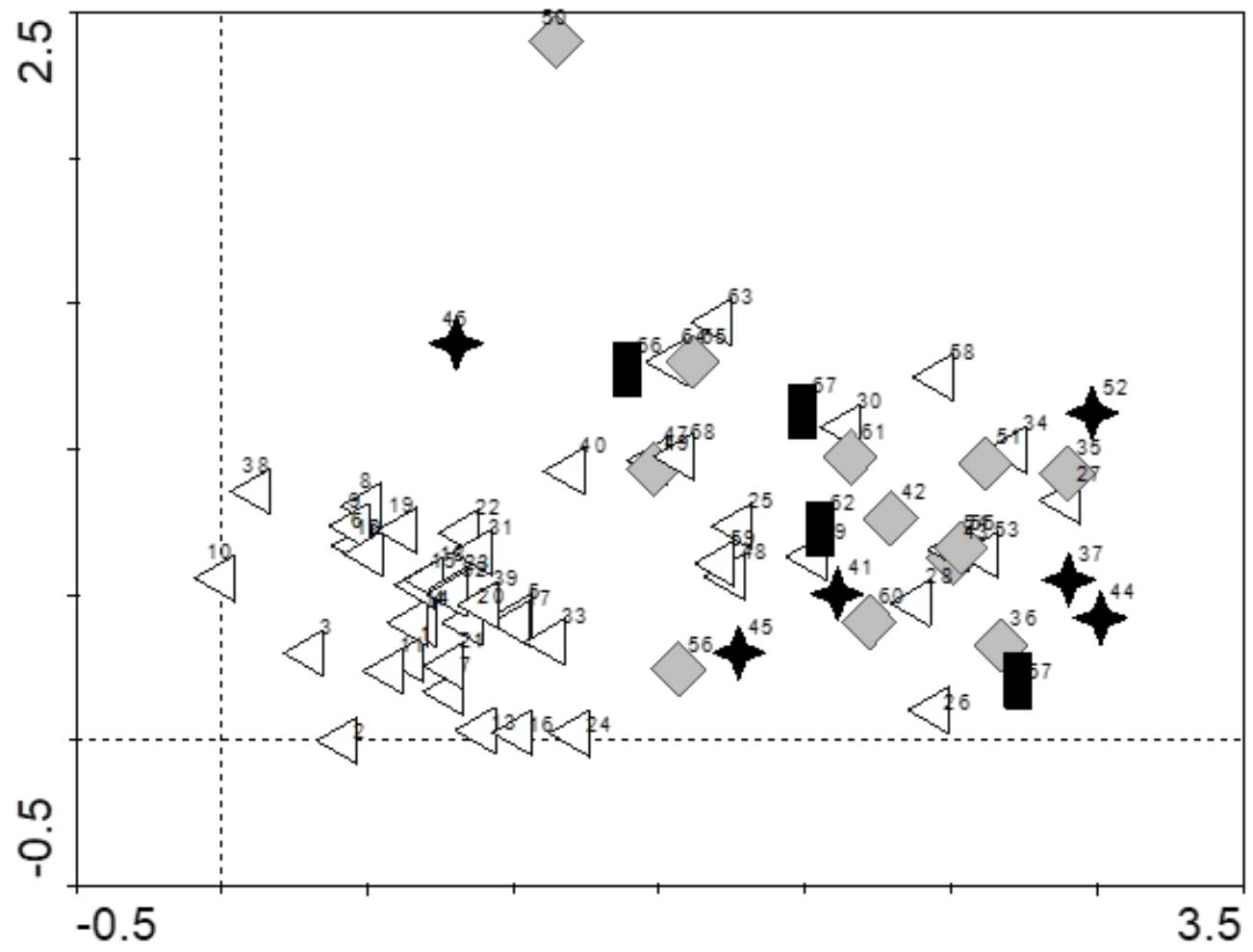

Figure 4: Diagramme de l'ordination des placettes dans le plan factoriel des axes 1 et 2 de l'Analyse Canonique des Correspondances Détendancées (DCA).
t: Culture
: Couloir
\: Pâture
Protection

\section{Analyse directe du gradient de distribution des espèces (CCA)}

L'analyse a été reprise avec les variables environnementales à travers une Analyse Canonique des Correspondances (ACC) (Figure 4). Cette dernière montre un regroupement des relevés au centre de l'ordination. Le tableau 2 montre les valeurs propres, la longueur des gradients et la variance expliquée par les quatre premiers axes de l'ACC. D'après ce tableau, les 4 premiers axes factoriels expliquent $11,9 \%$ de la variance totale. Cependant les axes 1 et 2 expliquent respectivement $6,1 \%$ et $2,6 \%$ la dispersion des nuages de point sur le plan de l'ordination (Figure 4). Pour mieux appréhender les facteurs écologiques qui influencent la moindre différence floristique, le test de permutation de Monte-Carlo a été réalisé sur les mêmes matrices. Le tableau 2 résume le résultat du test après 499 répétitions. Il montre que la pente et les cultures sont les plus fortement corrélées avec les relevés. 
Tableau 2: Valeurs propres, longueur des gradients et variance expliquée par les quatre premiers axes du DCA

\begin{tabular}{lccccc}
\hline Axes & 1 & 2 & 3 & 4 & Inertie totale \\
\hline Valeurs propres & 0,476 & 0,286 & 0,179 & 0,137 & 4,165 \\
Longueurs des gradients & 3,012 & 2,396 & 2,519 & 1,862 & \\
Pourcentage cumulatif de variance expliquée : & 11,4 & 18,3 & 22,6 & 25,9 & \\
\hline
\end{tabular}
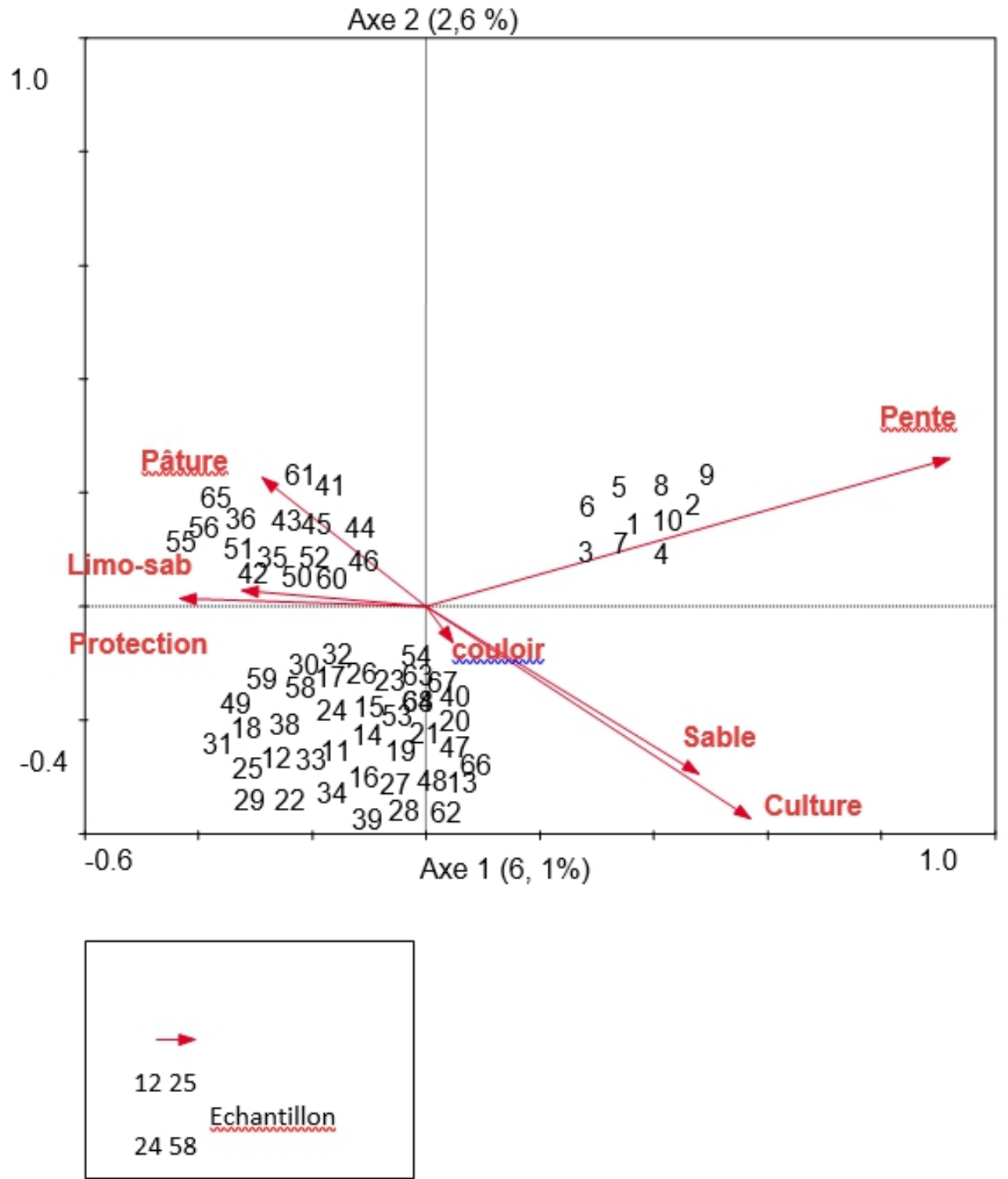

Figure 5 : Diagramme de l'ordination des placettes et des variables environnementales dans le plan factoriel des axes 1 et 2 de l'ACC (axes : 1 horizontal et 2 vertical) 


\section{Discussion}

L'analyse de la liste floristique globale a fait ressortir une dominance de Poaceae (16 espèces, soit 11,11 \%). Les Poacea sont une des familles d'espèces végétales les plus cosmopolites. Cette dominance dans le peuplement a été rapporté par beaucoup d'études au Niger notamment Danjimo (2000), Kaou et al (2017). La présence secondairement des familles des Mimosaceae et Caesalpiniaceae quand-à elles indiqueraient le caractère sec du milieu comme rapporté dans Saâdou (1990), Mahamane (2005) et Inoussa (2008) pour l'étude de la végétation au Niger. La présence des espèces comme A. indica, M. oleifera, E. camaldulensis, $P$. juliflora, A. occidentale, M. indica, $P$. guajava serait fort probable consécutive à une introduction humaine. Il ressort de l'analyse du comportement adaptatif des espèces telles que les thérophytes $(45,14 \%)$ qui dominent dans tous les types d'occupation des sols, suivis des phanérophytes notamment les microphanérophytes $(25,69 \%)$ et les mésophanérophytes $(7,64 \%)$. Cette prédominance traduit un environnement sec auquel appartient la zone d'étude. Aussi l'importance des microphanérophytes témoigne du caractère arbustif de la strate ligneuse (Mahamane, 2005 ; Thiombiano, 2005). Les autres formes de vie notamment les géophytes, les rhizomateux, les nanophanérophytes, les mégaphanérophytes et les hydrophytes ont été très peu représentées dans cette rôneraie, ce qui pourrait être expliqué par leurs difficultés de survivre dans ce milieu fortement anthropisé. De telles observations confirment celles de Kaou et al. (2017) travaillant sur la végétation dunaire au Sud-est du Niger. Du point de vue phytogéographique, les espèces Soudano-Zambésiennes dominent dans tous les types d'occupation des sols pour les spectres bruts et les espèces Guinéo-Congolaises-Soudano-Zambésiennes pour les spectres pondérés sauf dans les cultures sous parc à $F$. albida où le spectre pondéré est dominé par les espèces Soudano-Zimbésiennes-Saharo-sindiennes, mais on note une absence des espèces Soudano-Zimbésiennes-Saharo-sindiennesméditerranéennes. Cette flore est à dominante herbacée et caractéristique de milieux très diversifiés du compartiment Sud-sahélien central. Elle est constituée pour plus de la moitié des espèces, par des annuelles mais également par des espèces pérennes herbacées et ligneuses qui forment pendant la saison des pluies tout comme en saison sèche, un important tapis herbacé qui donne à la rôneraie sa physionomie. Les espèces à mode de reproduction végétative notamment les Balanitaceae, les Ceasalpiniaceae, les Combtretaceae, les Rhamnaceae, etc. représentent un atout pour revégétaliser certaines zones dénudées de la rôneraie.

Les résultats de ce présent travail montrent aussi l'importance des paramètres écologiques dans la distribution des plantes. Les modes d'exploitation et la flore à eux seuls ne permettent pas de discriminer clairement des groupes de relevés qui forment des groupements végétaux. 
Cela signifie que les différentes unités d'occupation des terres et d'utilisation des sols auraient presque la même composition floristique. La reprise de l'analyse avec les variables environnementales à travers une Analyse Canonique des Correspondances (ACC) a permis d'observer un regroupement des relevés au centre de l'ordination. Cela veut dire que la moindre différence floristique observée entre les unités d'occupations des sols pourrait être expliquée par les variables environnementales. Donc les modes d'exploitation de la rôneraie auraient peu d'effet sur la répartition des espèces végétales. Différents travaux ont utilisé les analyses multivariées (DCA, CCA) pour étudier l'effet des paramètres écologiques sur la végétation (Sinsin, 1993 ; Oumarou, 2003 ; Mahamane, 2005 ; Ouedraogo, 2009) et ont montré que la composition floristique, la structure et la distribution de la végétation varient avec les conditions du milieu. Sur l'éco-complexe, certaines variables peuvent être plus discriminantes que d'autres. Les résultats du test Monte Carlo montrent que la pente et les cultures sont les plus fortement corrélées avec les relevés. La présence dans la rôneraie d'espèces d'affinités biogéographiques diverses semble probablement liée à sa position géographique qui est son appartenance au compartiment sud-sahélien central.

\section{Conclusion}

Cette étude a permis de mettre au point les connaissances sur la phytodiversité et les paramètres agro-écologiques déterminant la distribution de la végétation dans la rôneraie de Dasga au Niger. La végétation est plus dominée par des espèces cosmopolites (Poaceae) et des espèces indicatrices du milieu sec. Des difficultés se posent à d'autres espèces de bien vouloir se développer du faite du caractère anthropisé de la rôneraie. Ce qui du reste est confirmé par la présence des espèces d'arbres fruitiers à intérêt alimentaire. La pente et les cultures ont été les variables les plus fortement corrélées à la distribution de la végétation de la rôneraie. Aussi, la rôneraie de Dasga apparaît comme un site de haute biodiversité en raison de la diversité des milieux dans une aire relativement réduite. À ce titre, les zones encore boisées et floristiquement riches doivent faire l'objet de plus d'attention. Elles doivent être épargnées du défrichage pour les champs et protégées contre les feux de brousse et le surpâturage. Les résultats de cette étude peuvent être importants dans le choix des espèces les plus adaptées à la géomorphologie et tenant en compte des besoins de la communauté locale dans le cadre de l'aménagement de cet écosystème.

\section{References:}

1. PAFAGE (Projet d'Appui à la Formation et d'Assistance en Gestion de l'Environnement), 2004. Le système d'Information sur les Forêts Classées du Niger. Document du travail, 51pp. 
2. Achard F., 2001.Végétation du terroir de Ticko (canton de TorodiNiger). Inventaire floristique et quelques relevés. $30 \mathrm{pp}$.

3. Bazi HI., 2009. Rôle des couloirs de passage dans la conservation de la biodiversité végétale dans la commune de Tanda. 63 pp.

4. Berhaut J., 1971, 1974, 1975, 1976, 1979. Flore illustrée du Sénégal. Dicotylédones, 6 tomes.

5. Berhaut J., 1967. Flore du Sénégal. $2^{\mathrm{e}}$ éd. Clairafrique. Dakar. 481 pp.

6. Braun-Blanquet J., 1932. Plant sociology. The study of plant communities. Ed. McGray Hill, New York, London, 439 pp.

7. Baina D., 2000. Contribution à l'étude floristique, écologique et phytosociologique de la forêt classée de Gourou Bassounga et des milieux cultivés adjacents. Thèse de doctorat, Université Abdou Moumouni de Niamey. $151 \mathrm{pp}$.

8. CNEDD, 1998. Etude de la vulnérabilité des formations forestières au changement et variabilité climatique. Rapport d'étude, 15 pp.

9. Fournier A., Yoni M. and Zombre P., 2000. Les jachères à Andropogon gayanus en savane soudanienne dans l'ouest du Burkina Faso: flore, structure, déterminants et fonction dans l'écosystème. Etudes flor. vég. Burkina Faso. Fonds Documentaire IRD, 5: 3-32

10. Gauch H.G. and Wentworth T.R., 1976. Canonical correlation analysis as an ordination technique. Vegetation 33:17 -22

11. Hamidou A., Boubé M., Ali M., Saadou M. and Abass I., 2012. Caractérisation de la dynamique spatio-temporelle de l'occupation des terres dans le complexe des forêts de classées de Dan kada Dodo - Dan Gado (région de Maradi, Niger). Journal des Sciences de l'Environnement 1 (1): 16-26

12. Hill MO. and Gauch HG., 1980. Detrended Correspondence Analysis, an improved ordination technique. Vegetation 42: 47-58.

13. Hutchinson J., Dalziel JM. and Keay R., 1958. Flora of West Tropical Africa. Crown Agents for oversea governments and adminstration, Milbank.London. 2nd edition, 828 pp.

14. Inoussa MM., 2008. Etude de la végétation d'un site de feux d'aménagement dans la reserve totale de faune de Tamou, Mémoire DEA, Université de Niamey, 82 pp.

15. Issiaka IM., Yamba B., Yansheng G., 2012. Land-use and land-cover change in semi-arid zone: the case of Waro-Souloulou Area in Goulbi Maradi Watershed in the Republic of Niger. Environ Nat Resou Res 2(1):47-62. https://doi.org/10.5539/enrr.v2n1p47

16. Kaou KAK., Manzo OL., Dan Guimbo I., Karim S., Habou R. et Paul R., 2017. Diversité floristique et structure de la végétation dans la zone dunaire du sud-est du Niger : Cas de Mainé soroa. Journal of Applied Biosciences 120: 12053-12066 
17. Larwanou M. and Saadou M., 2005. Biodiversity of ligneous species in semi-arid to arid zones of southwestern Niger according to anthropogenic and natural factors. Agriculture, Ecosystems and Environment 105: 267-271

18. Mahamane A., 2005. Etudes floristique, phytosociologie et phytogéographique de la végétation du Parc Régional du W du Niger. Thèse d'Etat, Univ. Libre de Bruxelles, p 497.

19. Mahamane A., Saâdou M, Bakasso Y., Abassa I., Aboubacar I. et Karim S., 2007. Analyse diachronique de 1'occupation des terres et caractéristiques de la végétation dans la commune de Gabi (région de Maradi, Niger). Sécheresse 18 (4): 296-304.

20. Morou B., 2010. Impacts de l'occupation des sols sur l'habitat de la girafe au Niger et enjeux pour la sauvegarde du dernier troupeau de girafes de l'Afrique de l'Ouest. Thèse de doctorat, Université Abdou Moumouni de Niamey, p 198.

21. Oumarou M., 2003. Etudes écologique, floristique, phytogéographique et phytosociologique des inselbergs du Bénin. Thèse de doctorat, Université Libre de Bruxelles, Faculté des Sciences, Laboratoire de Botanique Systématique, p 210.

22. Moussa M., Larwanou M., Saley K., Saadou M., 2016. Resilience to stress of woody species in Faidherbia albida (Del) A. Chev. and Prosopis africana (Guill., Perrot and Rich.) Taub. parklands in the Sahelian Niger. Journal of Biodiversity and Environmental Sciences (JBES) 8 (3): 107-124.

23. Palmer MW., 1993. Putting things in even better order: the advantages of canonical correspondence analysis. Ecology.74 (8): 2215-2230.

24. Peet R.K., Knox RG., Case JS. and Allen RB., 1988. Putting things in order: the advantages of detrended correspondence analysis. American Naturalist 131: 924-934.

25. Peyre de Fabregues B., 1972. Lexique de noms vernaculaires de plantes du Niger. $2^{\mathrm{e}}$ éd. $155 \mathrm{pp}$.

26. Roussel B., 1987. Les groupements végétaux hydrophiles et rupicoles d'une région sahélienne (l'Ader Doutchi, République du Niger). Doctorat ès Sciences naturelles, UFR de recherche scientifique et Technique, Université Blaise pascal de Clermont-Ferrand, 342 pp.

27. Saadou M., 1984. Contribution à l'étude de la flore et de la végétation des milieux drainés de l'ouest de la République du Niger, de la longitude de Dogondoutchi au fleuve Niger. Thèse de 3e cycle, Univ. de Bordeaux II, p 177.

28. Saadou M., 1990. La végétation des milieux drainés nigériens à l'est du fleuve Niger. Thèse de doctorat, Université Niamey, Niger, p 393. 
29. Sadda A-S., Diouf A., Lawali S., Ouedraogo M., Bogaert J. et Mahamane A., 2016. Pression anthropique et dynamique paysagère en zone rurale semi-aride : cas de la commune de Tibiri, région de Maradi (Niger). Tropicultura, 34 (2): 127-139.

30. Sinsin B., 1993. Phytosociologie, écologie, valeur pastorale, production et capacité de charge des pâturages naturels du périmètre Nikki-Kalalé au Nord-Bénin. Thèse de Docteur en Sciences Agronomiques. Université Libre de Bruxelles, 390 pp.

31. Soumana I., 2011. Groupements végétaux pâturés des parcours de la région de Zinder et stratégies d'exploitation développées par les éleveurs Uda'en. Thèse de Doctorat de l'Université de Niamey, 222 pp.

32. Soumana I., Mahamane A., Gandou Z., Ambouta J.M.K. and Saadou M., 2012.Vegetation and plant diversity pattern study of central eastern Niger grasslands. Int. J. Biol. Chem. Sci., 6 (1): 394-407.

33. TerBraak CJF., 1986.Canonical correspondence analysis: a new eigen vector technique for multivariate direct gradient analysis. Ecology, 67: 1167-1179.

34. TerBraak CJF. and Smilauer P., 1998. CANOCO Reference Manual and User's Guide to Canoco for Windows.Software for Canonical Community Ordination (version 4). Microcomputer Power (Ithaca, NYUSA), $352 \mathrm{pp}$.

35. White F., 1983. La végétation de l'Afrique. Mémoire accompagnant la carte de végétation de l'Afrique. Unesco / AETFAT / UNSO, ORSTOM / UNESC, 384 pp. 